PharmacoEconomics \& Outcomes News 874, p8 - 20 Mar 2021

\title{
Australians willing to accept short-term restrictions during pandemic
}

The Australian population appears to be willing to accept short-term lockdown restrictions to avoid health consequences during the COVID-19 pandemic, according to findings of a survey published in The Patient - PatientCentered Outcomes Research.

A discrete-choice experiment (DCE) with eight choice tasks was developed during April-May 2020 to investigate the acceptability of various infection control measures and how respondents considered the trade off between economic and health outcomes during the COVID-19 pandemic. Attributes of the DCE covered restriction control measures, duration of restrictions, tracking, numbers of COVID-19 infections and COVID-19-related deaths, job losses, government expenditure and personal tax levy.

The survey was completed by 1046 Australians, only $46 \%$ of whom reported that they had downloaded the COVIDSafe app.

Overall, respondents generally expressed preferences for policies that avoided a high infection-related death rate, but also considered lower unemployment and government expenditure were important. Although respondents preferred shorter duration restrictions, there was no significant variation in their preferences for differing levels of control measures. Respondents preferred the use of mobile phone tracking or bracelets compared with no tracking.

Latent class analysis identified two classes of people: Class 1 ( $57 \%$ of respondents) expressed preferences for the economy to remain open under some control measures such as the use of tracking bracelets, while Class $2(43 \%)$ expressed stronger preferences for policies that reduced avoidable deaths. Class 1 respondents significantly preferred medium-level restrictions to low-level restrictions, tracking bracelets to no tracking bracelets, and $5 \%$ or $3 \%$ tax levies versus a $1 \%$ levy, and thought 500000 deaths was significantly less acceptable than 10000 deaths.

"Class 1 were more likely to agree that social distancing, isolation and quarantine measures were excessive and that the impact of the restrictions were too costly, whereas Class 2 were more likely to strongly disagree with these two statements," said the investigators.

Manipis K, et al. Exploring the Trade-Off Between Economic and Health Outcomes During a Pandemic: A Discrete Choice Experiment of Lockdown Policies in Australia. The Patient - Patient-Centered Outcomes Research : 11 Mar 2021. Available from: URL: http://doi.org/10.1007/s40271-021-00503-5 